\title{
A Case of 5-Fluorouracil-Induced Coronary Artery Vasospasm in a Patient With Salivary Gland Cancer
} Iva N. Dimitrova ${ }^{1}$

1. Cardiology, St. Ekaterina University Hospital, Medical University of Sofia, Sofia, BGR

Corresponding author: Iva N. Dimitrova, dimytrova@yahoo.com

\begin{abstract}
5-Fluorouracil (5-FU), a pyrimidine analogue, is widely used in different chemotherapy regimens with established indications for the treatment of gastrointestinal, breast, head, and neck tumors. Various prospective studies including randomized controlled trials and retrospective reviews have shown a wide range of reported incidence of cardiotoxicity related to 5-FU use. This incidence is dependent on drug regimen, doses, concomitant therapy, patients' clinical characteristics, and risk factors. Herein, we present a clinical case of coronary vasospasm mimicking ST-elevation myocardial infarction during a 5-FU infusion for salivary gland cancer. Cardiologists and oncologists must keep in mind the potential life-threatening side effects of 5-FU on the heart and they must be familiar with the risk factors for their occurrence and their management strategies.
\end{abstract}

Categories: Cardiology, Emergency Medicine, Oncology

Keywords: 5-fluorouracil, myocardial infarction, coronary vasospasm, chemotherapy, salivary gland cancer

\section{Introduction}

Cancer is the second leading cause of death worldwide. The World Health Organization predicts that there will be a rise in cancer incidence by $70 \%$ over the next 20 years [1]. 5-Fluorouracil (5-FU) is a

chemotherapeutic drug used in the treatment of gastrointestinal, esophageal, renal, bladder, breast, head, and neck tumors. It can cause cardiotoxic effects including chest pain, myocardial infarction, arrhythmias, myocarditis, pericarditis, heart failure, and death [2]. The reported incidence is variable, between $0-20 \%$; the most frequent symptom is chest pain (0-18.6\%), reported mortality varies from 0 to $8 \%$ [3].

Possible factors increasing 5-FU cardiotoxicity are: underlying cardiovascular diseases, traditional risk factors like hypertension, hyperlipidemia, smoking; continuous vs bolus regimen of application; concomitant or previous radiation therapy; and simultaneous administration of another cardiotoxic drug $[2,4]$. Further investigations on risk stratification are needed because of the lack of definitive data and the controversial results of previous studies.

Review began 09/23/2020 Review ended 10/07/2020 Published 10/10/2020

\section{(๑) Copyright 2020}

Dimitrova. This is an open access article distributed under the terms of the Creative Commons Attribution License CC-BY 4.0., which permits unrestricted use, distribution, and reproduction in any medium, provided the original author and source are credited.
Suggested mechanisms of 5-FU toxicity are coronary vasospasm (endothelial or smooth muscle dysfunction), autoimmune-mediated injury of the myocardium, thrombogenic effects or thrombus formation, direct myocardial toxicity causing necrosis, accumulation of metabolites, altered red blood cell morphology, and impaired oxygen-carrying capacity $[2,5]$.

In this report we present a case of suggested coronary vasospasm, mimicking acute myocardial infarction in a 67-year-old man, during 5-FU infusion for salivary gland cancer.

\section{Case Presentation}

A 67-year-old man was transferred from the Oncology Department in our Cardiology Intensive Care Unit (ICU) with suspected ST-elevation myocardial infarction. He had a history of mucoepidermoid carcinoma of the left salivary gland (T2N1M0), excised with lymph node dissection and treated with radiotherapy eight months earlier. Because of cancer recurrence, a chemotherapy regimen was administered - 5-FU continuous intravenous infusion, cetuximab and cisplatin. On the third 5-FU application, during an infusion the patient suffered from continuous chest pain. The electrocardiogram (ECG) showed ST elevation on leads I, aVL, II, III, aVF, hyperacute T waves V2-V6 with reciprocal changes in aVR (Figure 1). 


\section{Cureus}

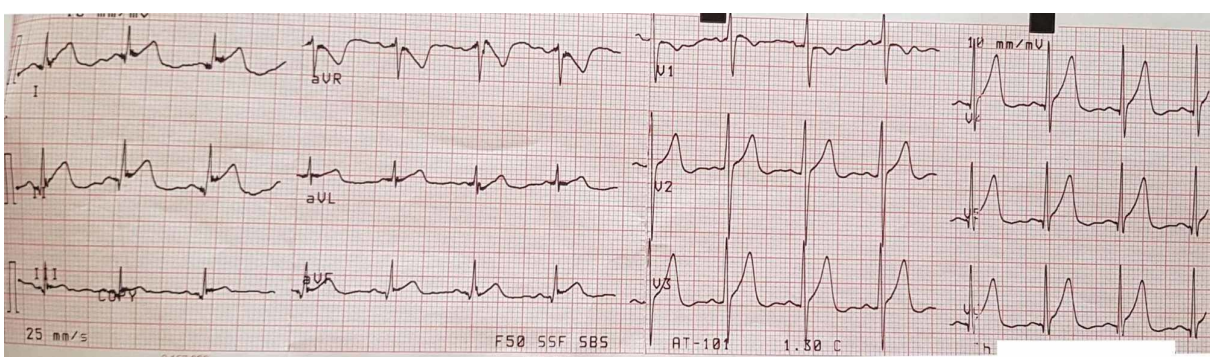

FIGURE 1: ST elevation on leads - I, aVL, II, III, aVF, hyperacute T waves V2-V6 with reciprocal changes in aVR during an anginal attack

The 5-FU infusion was discontinued, and sublingual nitroglycerin administered, with pain relief occurring after 10 minutes. In cardiology ICU ECG showed complete resolution of the ST changes observed earlier and there was complete pain relief (Figure 2).

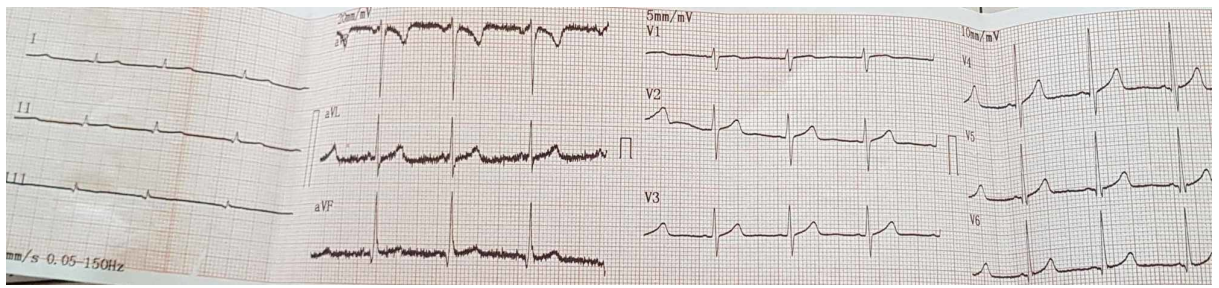

FIGURE 2: Complete resolution of the ST changes after sublingual nitroglycerin administration.

The patient had no history of heart disease. He had arterial hypertension, dyslipidemia, and smoking as risk factors. Samples for myocardial necrosis markers - creatine phosphokinase, creatine phosphokinase-MB and troponin $\mathrm{T}$ were within normal ranges. Although the echocardiography showed no wall motion abnormalities, preserved left ventricular ejection fraction, and mild mitral regurgitation, coronary angiography was performed which revealed non-obstructive coronary artery disease (Figure $3 \mathrm{a}$-c).
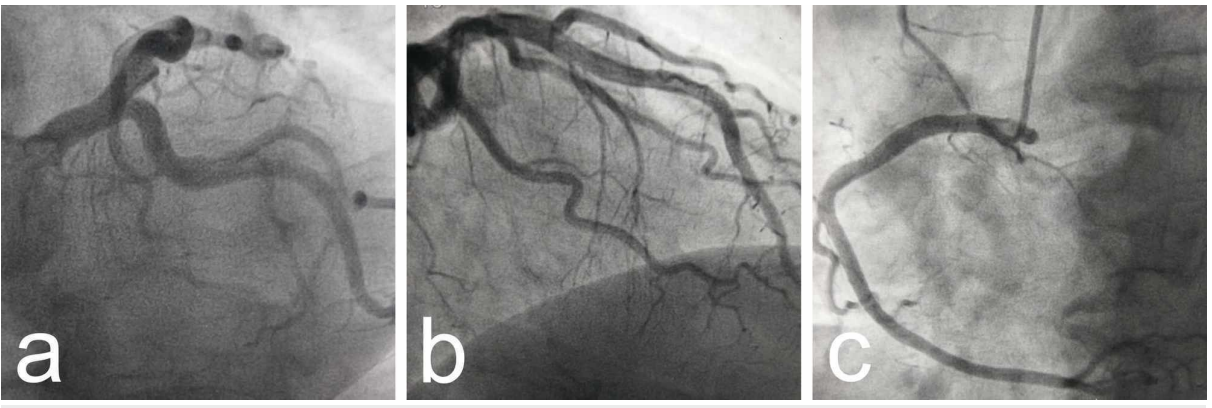

FIGURE 3: a, b) Normal appearance of left coronary system during angiography; c) Normal appearance of right coronary artery.

During hospital stay, the patient had no complaints, his ECG, cardiac enzyme levels, and echocardiography remained normal. Suggesting the relationship between 5-FU administration, typical chest pain, and reversible ECG changes probably due to coronary vasospasm in the context of chemotherapy cardiotoxicity, we advised the oncologists to consider therapeutic regimen modification, if not possible - strict ECG monitoring during the 5-FU infusion and prophylactic use of calcium channel blocker (CCB) and nitrate. The patient was discharged on the fourth day after the procedure.

\section{Discussion}

Cardiovascular diseases and cancer are a leading cause of morbidity and mortality worldwide. Cardiotoxicity is an important and sometimes life-threatening effect of some chemotherapeutic drugs. 5-FU is in wide use for the treatment of solid tumors and is the second most cardiotoxic chemotherapeutic drug [6]. The 
reported wide range of its incidence, the controversial risk profile reported in studies, the numerous proposed mechanisms of 5-FU-induced cardiotoxicity, and the lack of established guidelines for treatment define the need for further research.

Coronary vasospasm is the most established theory for 5-FU-related cardiotoxicity. Despite the evidence of significant ST changes and, sometimes, elevated troponin levels, often no coronary artery disease is observed in coronary angiography or computed tomography [7-9]. Coronary artery and brachial artery vasospasm following 5-FU infusion has been observed in angiography or ultrasound [10-12]. Nitrates and CCB are effective in pain relief and seem to correct ECG changes [5]. This data taken together supports the vasospastic theory, although other pathophysiological mechanisms of 5-FU-induced cardiotoxicity are possible.

In the presented case there were diffuse ECG changes during the pain period that may be a sign of pervasive coronary artery vasospasm rather than vasospasm of an isolated epicardial artery. The discontinuation of 5FU infusion and the application of sublingual nitroglycerine fully relieved the chest pain and reversed the ECG and echocardiography changes. Management approaches have been suggested for 5-FU induced cardiotoxicity. It is recommended to discontinue 5-FU application or if not possible, rechallenge carefully the treatment with administration of CCB, long-acting nitrates, and close cardiac monitoring [2]. Experimental treatments exploring the protective effect of coenzyme complex or glucagon-like peptide-1 analogues may reveal new options in the management of 5-FU-induced cardiotoxicity [4].

\section{Conclusions}

Clinical manifestations of 5-FU-induced cardiotoxicity are often treatable and reversible but could be lifethreatening. This requires patient risk stratification, knowledge of the potential mechanisms, and management strategies. Close collaboration between cardiologists and oncologists is advisable in order to achieve improved clinical outcomes and to reduce potential complications.

\section{Additional Information \\ Disclosures}

Human subjects: Consent was obtained by all participants in this study. Conflicts of interest: In compliance with the ICMJE uniform disclosure form, all authors declare the following: Payment/services info: All authors have declared that no financial support was received from any organization for the submitted work. Financial relationships: All authors have declared that they have no financial relationships at present or within the previous three years with any organizations that might have an interest in the submitted work. Other relationships: All authors have declared that there are no other relationships or activities that could appear to have influenced the submitted work.

\section{Acknowledgements}

The author thanks Assist. Prof. Dr. Svetoslav A. Slavchev (Department of Orthopedics and Traumatology, Medical University of Sofia, Bulgaria) for his kind proofreading of the English text.

\section{References}

1. Cancer. (2018). Accessed: May 1, 2019: https://www.who.int/news-room/fact-sheets/detail/cancer .

2. Sara JD, Kaur J, Khodadadi R, et al.: 5-fluorouracil and cardiotoxicity: a review . Ther Adv Med Oncol. 2018, $10.1177 / 1758835918780140$

3. Polk A, Vaage-Nilsen M, Vistisen K, Nielsen DL: Cardiotoxicity in cancer patients treated with 5fluorouracil or capecitabine: a systematic review of incidence, manifestations and predisposing factors. Cancer Treat Rev. 2013, 39:974-84. 10.1016/j.ctrv.2013.03.005

4. Chong JH, Ghosh AK: Coronary artery vasospasm induced by 5-fluorouracil: proposed mechanisms, existing management options and future directions. Interv Cardiol. 2019, 14:89-94. 10.15420/icr.2019.12

5. Sorrentino MF, Kim J, Foderaro AE, Truesdell AG: 5-fluorouracil induced cardiotoxicity: review of the literature. Cardiol J. 2012, 19:453-458. 10.5603/cj.2012.0084

6. Anand AJ: Fluorouracil cardiotoxicity. Ann Pharmacother. 1994, 28:374-378. 10.1177/106002809402800314

7. Tajik R, Saadat H, Taherkhani M, Movahed MR: Angina induced by 5-fluorouracil infusion in a patient with normal coronaries. Am Heart Hosp J. 2010, 8:111-112. 10.15420/ahhj.2010.8.2.111

8. Atar A, Korkmaz ME, Ozin B: Two cases of coronary vasospasm induced by 5-fluorouracil . Anadolu Kardiyol Derg. 2010, 10:461-462. 10.5152/akd.2010.147

9. Connolly S, Scott P, Cochrane D, Harte R: A case report of 5-fluorouracil-induced coronary artery vasospasm. Ulster Med J. 2010, 79:135-136.

10. Luwaert RJ, Descamps O, Majois F, Chaudron JM, Beauduin M: Coronary artery spasm induced by 5fluorouracil. Eur Heart J. 1991, 12:468-470. 10.1093/oxfordjournals.eurheartj.a059919

11. Shoemaker LK, Arora U, Rocha Lima CM: 5-fluorouracil-induced coronary vasospasm. Cancer Control. 2004, 11:46-49. 10.1177\%2F107327480401100107

12. Südhoff T, Enderle MD, Pahlke M, Petz C, Teschendorf C, Graeven U, Schmiegel W: 5-Fluorouracil induces arterial vasocontractions. Ann Oncol. 2004, 15:661-664. 10.1093/annonc/mdh150 